\title{
Article \\ Articulating Resilience: Adaptive Locomotion of Wheeled Tensegrity Robot
}

\author{
Tianyuan Wang *(D), Mark A. Post $(D)$ and Andy M. Tyrrell (D) \\ Department of Electronic Engineering, University of York, Heslington, York YO10 5DD, UK; \\ mark.post@york.ac.uk (M.A.P.); andy.tyrrell@york.ac.uk (A.M.T.) \\ * Correspondence: tw1356@york.ac.uk
}

Citation: Wang, T.; Post, M.A.; Tyrrell, A.M. Articulating Resilience: Adaptive Locomotion of Wheeled Tensegrity Robot. Electronics 2022, 11, 666. https://doi.org/10.3390/ electronics11040666

Academic Editor: Dah-Jye Lee

Received: 23 January 2022

Accepted: 19 February 2022

Published: 21 February 2022

Publisher's Note: MDPI stays neutral with regard to jurisdictional claims in published maps and institutional affiliations.

Copyright: (c) 2022 by the authors. Licensee MDPI, Basel, Switzerland. This article is an open access article distributed under the terms and conditions of the Creative Commons Attribution (CC BY) license (https:// creativecommons.org/licenses/by/ $4.0 /)$.

\begin{abstract}
Resilience plays an important role in improving robustness for robots in harsh environments such as planetary exploration and unstructured terrains. As a naturally compliant structure, tensegrity presents advantageous flexibility for enhancing resilience in robotic applications according to existing research. However, tensegrity robots to date are normally based on monolithic morphologies and are slow in locomotion. In this paper, we demonstrate how we adopt such flexibility to improve the robustness of wheeled robots by articulating modules with tensegrity mechanisms. The test results reveal the robot is resistant and resilient to external hazards in a fully passive manner owing to the continuous elasticity in the structure network. It possesses a good number of DoFs and can adapt to various terrains easily either with actuation or not. The robot is also capable of crawling locomotion aside from wheeled locomotion to traverse uneven surfaces and provide self-recovery from rollovers. It demonstrates good robustness and mobility at the same time compared with existing tensegrity robots and shows the competitiveness with conventional rigid robots in harsh scenarios. The proposed robot presents the capability of tensegrity robots with resilience, robustness, agility, and mobility without compromise. In a broader perspective, it widens the potential of tensegrity robots in practical applications.
\end{abstract}

Keywords: tensegrity; robot; resilience; articulation; modular; locomotion

\section{Introduction}

Environmental hazards and disturbances are widespread and long-standing challenges for many robot applications such as in planetary exploration, post-disaster rescue, and plant inspection [1-3]. They can arise from various sources-complex terrains, extreme weather conditions, or even human factors. Robots operating in such scenarios require robustness and resilience to withstand and potentially recover from such challenges and to complete their missions [4]. Over the last few years, soft robots have gained particular attention for their flexibility and compliance as well as potentially improved safety properties over conventional rigid robots [5]. Although they are exceptionally resilient, these robots are to some extent too "soft" to complete some tasks, for instance, lifting or carrying relatively heavy loads, as they normally do not have skeletons built inside the body to bear the weight [5]. Additionally, it is difficult to make them self-contained while retaining specified performance as they require cumbersome external actuation sources and equipment that are stationary and cannot move with the robot body [6]. Therefore, they are often implemented as manipulators or limbs as a part of a larger robot system [7-11]. Balancing these two concepts, research in tensegrity robots is growing owing to their unique characteristics [12]. Tensegrity robots are naturally compliant to external loads while retaining certain stiffness, that is, being soft and rigid at the same time. This characteristic is critical and competitive for the harsh scenarios mentioned above.

Tensegrity systems are visually non-intuitive. They are composed of discontinuous rigid elements in compression and a continuous network of elastic elements that are in tension [13]. They therefore possess many characteristics including stability, structural 
efficiency, reconfigurability, failure tolerance, deployability, and ease of modelling [14,15]. Research on tensegrity robotics initially explored morphologies and expanding the scale of static tensegrity structures through transforming the topology, a process called form finding [16]. From the 2010s, studies on applying a tensegrity structure to robots have increased as its potential with regard to the dynamics of the system was identified. Taking advantage of the compliance of the tensegrity icosahedron, the SUPERball series robots are capable of planetary landing on their own outer structures [17-22]. Kim et al. also considered the landing function. Their tensegrity robot can hop repetitively, using thrusters, up to $60 \mathrm{~m}$ high for travelling a long distance efficiently on the Moon [23]. Additionally, researchers also integrate tensegrity into aerial vehicles to protect from collision [24].

Existing tensegrity robots are normally based on absolute tensegrity structures. The morphologies include the icosahedron (the three introduced above), prism [25], spine [26], and tetrahedron [27]. These structures make good use of the characteristics of tensegrity but also introduce common limitations of such irregular structures. Unlike the typical forward process of designing from a tensegrity structure to a robot, here, the reverse, from robot demands to tensegrity, is required. Modern rigid robots have been developed over many years and many iterations and hold many well-proven features. How to further and integrate the advantages of tensegrity to a complete and comprehensive system, and to carry out practical tasks, are important avenues of research if tensegrity robots are to compete in real-world applications.

This paper presents a novel tensegrity robot design that adopts resilience as well as other advantages of tensegrity for harsh environments by articulating rigid wheeled robot modules within the tensegrity structure. The reason for using such an articulation structure is introduced in the subsequent section. Then, the proposed robot is tested in terms of its resilience from different perspectives. The results are then demonstrated to support the expectations of the new structure and discussed in Sections 4 and 5, followed by the conclusion in the final section.

\section{Motivation and Background}

The nature of tensegrity makes it an ideal structure for robots that require resilience. Tensegrity robots with high robustness and flexibility are normally based on a monolithic structure since the structure needs to be a continuous network of elements. However, it turns out they share some common shortcomings [28,29].

The first is slow terrestrial locomotion. Tensegrity robots can adopt several terrestrial locomotion approaches, such as widely used rolling [22,30] and crawling [26,31], as well as walking [32] and vibration [28,33]. However, none of these is fast compared with conventional rigid robots since any actuation-induced force change in one element will distribute across the structure. Emerging research focuses on speeding up the locomotion by improving the gait-using, for example, coevolutionary algorithms, central pattern generator (CPG), and reservoir computing [34]. Alternative approaches were also considered such as hopping and aerial locomotion, mentioned in Section 1. However, these do not address the root cause of the issue, hence the efficiency is not satisfactory.

The second challenge is the payload mounting. The irregular shape of the available space for payloads and the potential change in orientation make installation of payloads difficult. The tensegrity icosahedron is the one structure that has a relatively large convex polyhedron in its centre. There are two approaches to install the rigid payloads on a tensegrity structure [35]. However, to attach payloads through soft transition will bring in extra complexity while hard transition will reduce the performance provided by the tensegrity structure.

The third challenge is self-reconfigurability. Reconfigurability endows many capabilities to robots. This includes resilience. With various potential configurations, a number of issues can be mitigated by this reconfiguration. Tensegrity robots are naturally modular and reconfigurable, but it is difficult to make them self-reconfigurable. The lack of mobility of single elements, irregular networks of connections, and placement of elements introduce additional complexity to implement run-time reconfiguration of typical tensegrity robots. 
To summarise the challenges, what is required is a tensegrity robot that is resilient for challenging missions, but does not lose these fundamental capabilities and high level of potential for resilience. This paper reports on a novel method of using a versatile tensegrity structure to articulate a wheeled robot. Wheeled locomotion is a well-tried and generally appropriate method for many robot applications, while legged locomotion is rapidly developing [36]. With actuated articulation, the robot modules can be reconfigured to take wheeled, legged, and other forms for uncompromising functionality. The articulation adopts a tensegrity mechanism that is proposed in previous research [37]. It focuses on improving the motion efficiency and manoeuvrability. The mechanism is composed of passive and active tensegrity sub-structures. It has three degrees of rotational freedom and can operate either passively or actively. Owing to the flexibility of elastic cables and separation of rigid elements, the mechanism is compliant and resilient to disturbances. The proposed robot is shown in Figure 1 in a neutral posture of one possible configuration.

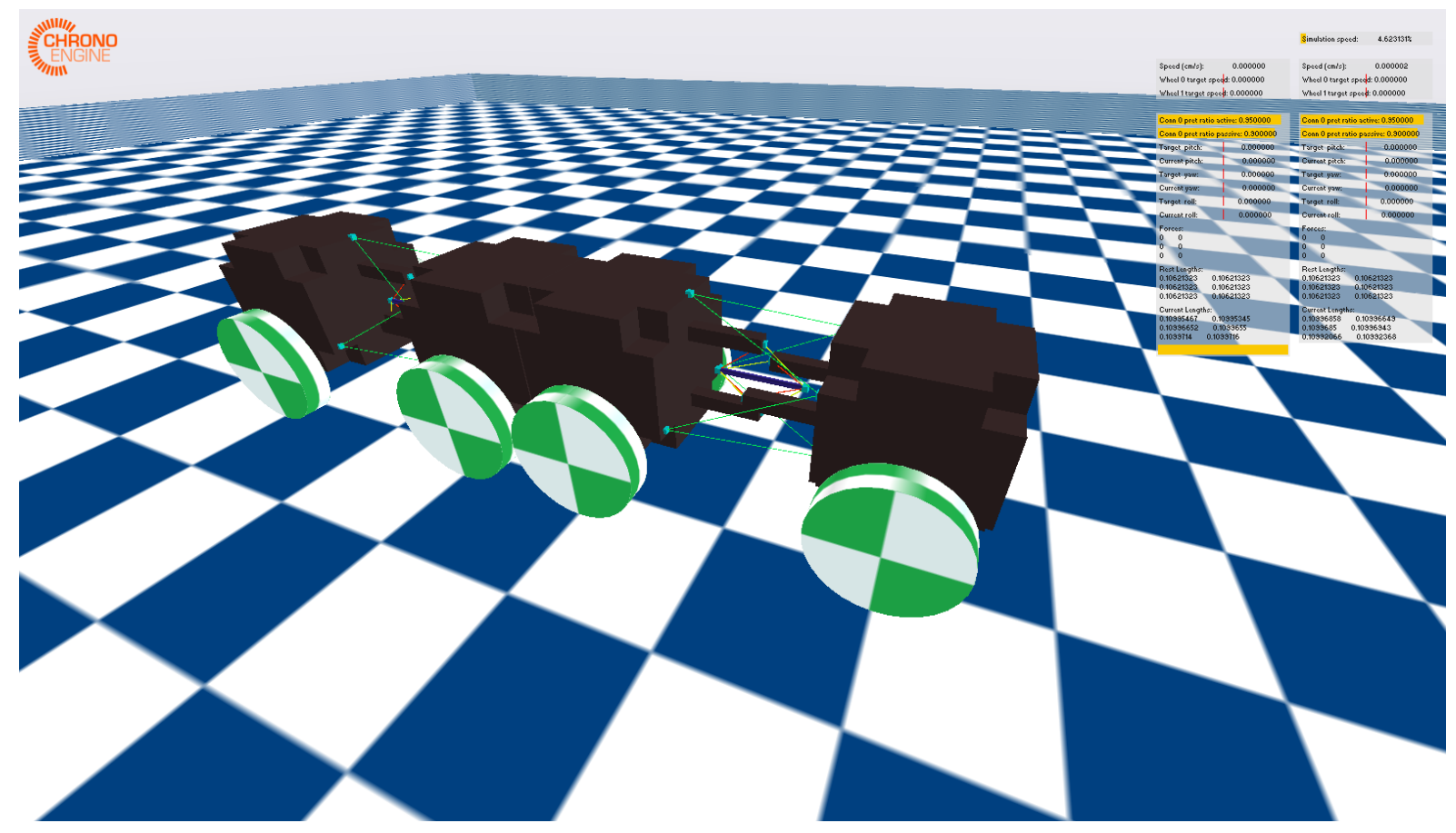

Figure 1. One configuration of the wheeled tensegrity modular robot, consisting of four modules with two wheels each and two tensegrity mechanisms.

\section{Materials and Methods}

Resilience of robots can be affected by different factors. This can be by hardware or software (or a combination of both), realised either structurally or systematically by means of control or configuration [38,39]. It generally refers to the robots' capability to recover from a damaged, hazardous, or deviated status [40]. For the proposed wheeled tensegrity modular robot, introducing tensegrity articulation enhances its resilience through both approaches. From the perspective of structure, the robot is expected to withstand environmental disturbances through absorbing energy with the elastic tensegrity network. From the perspective of locomotion, the robot is expected to switch between different locomotion approaches to adapt to terrains and scenarios. To comprehensively demonstrate such characteristics of the robot, the work reported in this paper tested them in a number of scenarios including evaluation of the natural frequency, static load, external impact resistance, fall off resistance, passive and active terrain adaptation, crawling, and rollover self-righting.

The tests outlined in this paper are based on the Chrono Engine simulation platform since it supports multibody physics and FEA-based cable objects at the same time. All rigid bodies in the simulation share the same and consistent properties, of which the restitution 
coefficient is 0.2 , the friction coefficient is 0.5 , and density is ABS $1.07 \mathrm{~g} / \mathrm{cm}^{3}$. The cables share the same Young's modulus of $2.7 \mathrm{GPa}$ and density of $1.15 \mathrm{~g} / \mathrm{cm}^{3}$ of Nylon. The diameter of active cables is $0.707 \mathrm{~mm}$ and $0.5 \mathrm{~mm}$ for passive cables, making the former have twice the cross-sectional area.

The tensegrity robot is created with primitive shapes. Each module body consists of 6 cuboid tiles and is consistent with the design of the sub-modular system [41]. The tensegrity mechanism uses 3 tiles on each module. The rods and wheels are created with cylinder shapes. Throughout all simulation tests, the passive cables maintain a constant pretension ratio of $90 \%$. For active cables, a series of pretension ratios from $90 \%$ to $99 \%$ with step size $1 \%$ are swept as per demands of the test scene.

\subsection{Integration of Resilience into Robots}

The proposed tensegrity robot has two key features which are the soft articulation based on the tensegrity mechanism and the rigid concatenation based on a coupling system. The former and the latter introduce flexibility and reconfigurability to the system, respectively, and together they endow resilience to the system.

\subsubsection{Tensegrity Articulation of Robot Modules}

The minimum independently functional unit of the robot consists of two wheeled modules and one tensegrity mechanism, which are referred to here as the robot assembly. Oscillation is one of the common properties of tensegrity structures, as well as other flexible systems. Such a system should avoid operating under external oscillating sources at its resonance frequencies. On the other hand, its flexibility also indicates compliance and resistance to external loads. Therefore, knowledge of the natural frequency and static resistance of the robot is essential for robust operation.

The natural frequency of the tensegrity robot presented here was obtained by analysing the oscillation of the free moving module under Earth surface gravity while the other module of the robot unit was fixed with respect to the world. The oscillation was generated by stressing the mechanism rapidly from a loose state to act as an impulse applied to the system.

As for the static resistance test, a slowly linearly increasing force/torque to the freemoving module along its $x, y$, and $z$ axes, corresponding to the roll, yaw, and pitch motion, with zero gravity, was applied, and the resultant angles of rotation were measured.

\subsubsection{Concatenation of Multiple Robot Assemblies}

Owing to the modular design, the robot structure can expand to different topologies by concatenating multiple robot assemblies using a rigid coupling system. It can be configured in various morphologies to meet the requirements of specific tasks and scenarios. In extreme and unknown environments, it is possible for robots to experience transient external impacts or to fall from heights. Therefore, the structure illustrated in this paper is a simple concatenation of two assemblies in series to investigate the resilience of such a minimal multiassembly configuration, that is four modules in total. For the two test scenes, the absolute translational acceleration of each module was recorded, and compared with a rigidly articulated replicate.

The impact test was performed by launching a solid metal ball along the axial axis towards the robot. The solid ball had a diameter of $40 \mathrm{~mm}$, a density of $8.05 \mathrm{~kg} / \mathrm{m}^{3}$, and was launched at $10 \mathrm{~m} / \mathrm{s}$ velocity.

The fall off test was conducted by making the robot roll freely down a slope and fall off onto a lower level. To obtain the same collision velocity, the robot was dropped from a fixed height of $0.2 \mathrm{~m}$ above and parallel with the slope. The angle of the slope was $30^{\circ}$ and the distance of the acceleration stage on the slope was $0.5 \mathrm{~m}$. The vertical distance from the edge of the slope to the lower ground was $1 \mathrm{~m}$. 


\subsection{Terrain Adaptation during Locomotion}

When coping with very rugged terrain or obstacles, it is very likely for robots to get stuck or trapped during locomotion. These challenges can be potentially overcome by improving the wheels' fit to the surface, implementing alternative locomotion approaches, or self-recovery from failure.

\subsubsection{Passive Adaptation on Uneven Terrain}

Due to the dual sub-structure design, the tensegrity mechanism does not lose its structural integrity when the active cables are in a loose state, that is, it can operate either passively or actively. When in passive mode, the articulation will not generate antagonistic forces for motions within its workspace. This greatly increases the workspace of wheels, which is similar to extending the stroke of a suspension system without introducing one. This is beneficial for challenging environments as the ground is rarely a flat surface. Wheels without enough workspace can cause poor contact with the terrain and lead to balancing and stability issues in robot locomotion. The passive adaptation can make the robot fit better to the surface to prevent or recover from unbalanced or stuck status.

To compare the performance of the tensegrity robot with a rigid articulated one, an uneven terrain is generated that consists of rectangular blocks of pseudorandom height using the same seed. The contact force on each wheel is recorded for analysis.

\subsubsection{Active Adaptation to Obstacles}

For situations that need to traverse more complex terrains, the actuated tensegrity mechanism can help the robot actively surmount unavoidable obstacles. To realise this active surmounting, the minimum 2-assembly configuration is just enough to guarantee enough mass of the stationary modules when moving free modules.

The obstacle is represented by a simple step placed in the path of locomotion. Assuming the robot has knowledge of the distance to the step, the step-climbing procedure is a predefined "pull-push" motion sequence based on this distance information. Firstly, the front module will pitch up before reaching the step. After the front axle passes the step edge, the front module will pitch down to pull the robot upwards. Secondly, the rear module will pitch down after the second axle passes the step edge to push the robot up further and at the same time the front module will return to the neutral posture. Finally, the rear module will return to the neutral posture after the third axle passes the step edge. A test run is recorded as a success if the fourth axle passes the step edge. The maximum step height is obtained by sweeping from $50 \mathrm{~mm}$ upwards with step size $10 \mathrm{~mm}$ and then $1 \mathrm{~mm}$ from the maximum result of the $10 \mathrm{~mm}$ sweep.

\subsubsection{Traversing with Crawling Locomotion}

While wheeled locomotion is suitable for relatively continuous surfaces, legged locomotion is a good choice for scattered and discrete terrains which cannot be overcome by the former. Owing to the three degrees of rotational freedom in each joint, the robot proposed in this paper is capable of imitating the crawling locomotion of multiped creatures without actuated legs. However, due to the symmetry of the spine-based gait, a minimum of 6 feet are required for stable contact with the ground for the feet in stance phase. For the proposed robot, the wheels attached to the middle two modules are rigidly concatenated and can therefore act as one pair of "feet", resulting in 3 feet pairs in total. 
To improve the resilience of the crawling locomotion to disturbance, a central pattern generator is applied to control the gait. The oscillator of the CPG is based on the model proposed by Ijspeert et al., and given as follows [42]:

$$
\begin{aligned}
\dot{\theta} & =2 \pi v_{i}+\sum_{j} r_{j} w_{i j} \sin \left(\theta_{j}-\theta_{i}-\phi_{i j}\right) \\
\ddot{r}_{i} & =a_{i}\left(\frac{a_{i}}{4}\left(R_{i}-r_{i}\right)-\dot{r}_{i}\right) \\
x_{i} & =r_{i}\left(1+\cos \left(\theta_{i}\right)\right)
\end{aligned}
$$

where $\theta$ and $r$ denote the actual phase and amplitude of the oscillators. $w$ and $\phi$ denote the weight and phase bias of the oscillators' couplings. In this case, the intrinsic frequency $v$ and amplitude $R$ are set to $0.6 \mathrm{~Hz}$ and 0.25 , respectively, to allow the robot to crawl straight ahead. The constant $a$ controls how quickly the oscillator converges to intrinsic amplitude and is set to 50 .

The CPG network consists of 8 nodes and each node pair controls one degree of freedom of the robot. Since the pitch motion does not contribute to the rhythmic motion during crawling, the 4 node pairs control the yaw and roll motion of each assembly, respectively, arranged as illustrated in Figure 2.
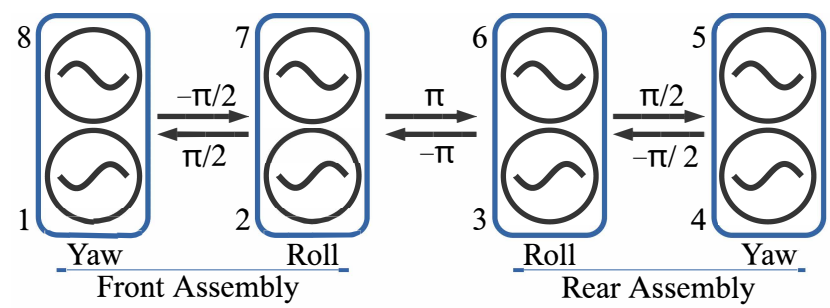

Figure 2. Structure of the CPG network generating a crawling gait for the 2-assembly tensegrity robot.

The roll motion of each assembly leads the yaw motion by $\pi / 2$. The adjacent two assemblies are shifted by one $\pi$ to create symmetrical motion on odd and even feet. The two oscillators in each node pair are anti-phase and subtracted to generate bipolar output as the oscillator output $x$ is always a positive value.

\subsubsection{Self-Righting}

In extreme and unpredictable environments, robots can get into unrecoverable states, such as rollover, which can eventually lead to a complete failure of missions. In this case, a scalable and reconfigurable modular robot composed of multiple assemblies will be able to recover from such situations by manipulating its "limbs" like human beings. Therefore, the robot structure was tested for self-righting capability for the minimum 2-assembly configuration. The robot was initially placed sideways on the ground. The self-righting procedure was completed by a predefined motion sequence. The orientation of each module was recorded.

\section{Results}

\subsection{Structural Resilience}

To visualise the oscillation of the robot, the length of the active cable connecting the front top and rear left nodes was chosen as shown in Figure 3. At the very beginning, due to the rapid stressing, the cable vibrates and then enters the harmonic motion stage. Since the two modules become closer due to a small displacement caused by pretension, the waveform eventually converges to different stable lengths corresponding to the pretension ratios. The rate that oscillations fade away gradually decreases along with the decrease in the pretension amount, while the overall fading rate is slow, indicating a large $Q$ factor and frequency rejection band. From Figure 3, it can be seen that different pretension ratios do not obviously affect the natural frequency of the mechanism. The $90 \%$ pretension ratio leads 
to a natural frequency of $14.9 \mathrm{~Hz}$ and a $99 \%$ pretension ratio leads to $15.9 \mathrm{~Hz}$, which gives an overall difference of $1 \mathrm{~Hz}$ and an approximately $0.11 \mathrm{~Hz}$ average difference between two adjacent samples.

Spectrum of Cable Length

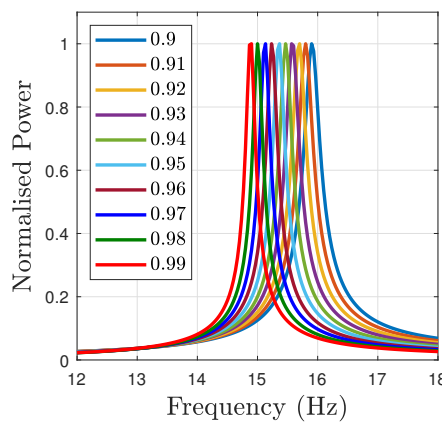

Waveform of Cable Length

Active, Front Top - Rear Left

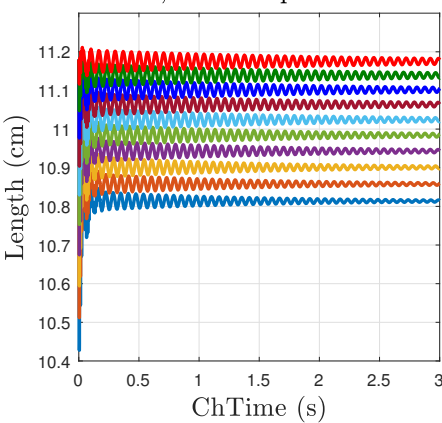

Figure 3. Normalised spectrum of the oscillation and the waveform of one cable length. Left: The spectrum showing the natural frequency of the robot assembly in a neutral position. Right: The length waveform of the cable connecting front top and rear left nodes for $3 \mathrm{~s}$ from the time of sudden stressing.

The static load results reveal a greater effect of pretension ratios than natural frequencies, and clearly demonstrate the compliance, resistance, and resilience to external loads in a fully passive manner. To eliminate the interference from other degrees of freedom, constraints relative to each test motion are added-coaxial constraint for roll motion, coplanar constraint for pitch and yaw motions. According to the results illustrated in Figure 4, the rotation-force/torque curves share a common characteristic that after crossing a smooth region, during which the deformation of the robot assembly gradually increases as the force accumulates, they then enter a rapid growth stage up to the physical limitation of the mechanism. As for the differences, an obvious one is that the yaw and roll motion tests can recover from over-deformed states but not the pitch motion. For the pitch motion, the robot assembly gets into unrecoverable states with the final force of approximately $34.17 \mathrm{~N}$ and $55.98 \mathrm{~N}$ for $99 \%$ and $90 \%$ pretension ratios, respectively. For the yaw motion's case, the values are approximately $27.36 \mathrm{~N}$ and $49.27 \mathrm{~N}$, and start to recover at a small load of around $24.22 \mathrm{~N}$ and $13.10 \mathrm{~N}$, causing obvious vibrations. Since the physical constraint of the roll motion does not allow it to deform significantly, the recovery curves almost overlap with the deform ones while the former contains slight vibrations at the start of the recovery stage. The forces are approximately $5.52 \mathrm{~N}$ and $9.83 \mathrm{~N}$ for $99 \%$ and $90 \%$ pretension ratios. The intermediate pretension ratios' results separate evenly among these two extremes.

The impact test results are shown in Figure 5a. The four modules of the robot are numbered 00, 01, 10, and 11, where the left digit represents the robot assembly and the right digit represents the module in the assembly. Module 11 is the one directly facing the ball. Data are taken for $0.2 \mathrm{~s}$ starting from the time of contact. The rigid robot's result is the data set labelled 0 pretension ratio. The maximum acceleration of its four modules are almost the same as expected since it is rigidly articulated. Module 11 of the tensegrity robot bears about two times higher maximum acceleration than the rigid one. In contrast, the rest of the modules of the tensegrity robot hold much lower maximum acceleration at about $1 / 3$. This proves that the tensegrity articulation prevents the impact from passing unattenuated through the whole network of the robot system. It can be interpreted as a sacrifice by the module of impact to protect the rest of the robot from externally inflicted damage. The robot can then decouple the incapacitated assembly and recover from malfunction. 
Pitch - $z$ Axis Force

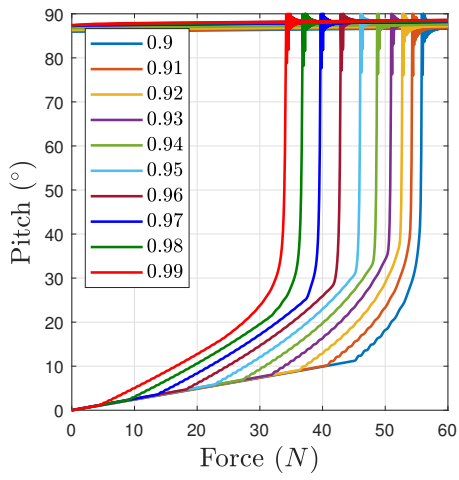

Yaw - $y$ Axis Force

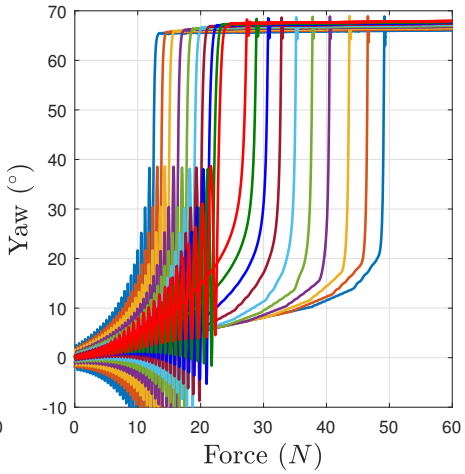

Roll - $x$ Axis Torque

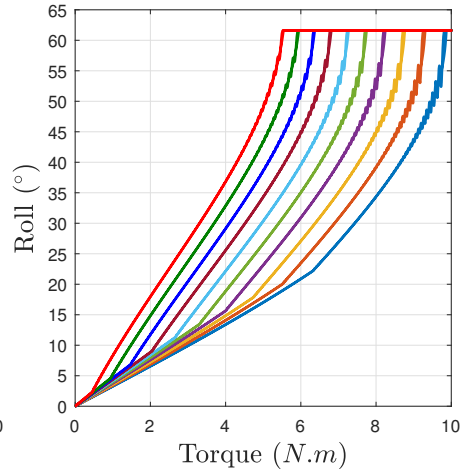

Figure 4. The rotation caused by the force or torque applied on the free-moving module. (Left): The pitch motion caused by applying the force along the $z$ axis of the free-moving module. (Middle): The yaw motion caused by applying the force along the $y$ axis of the free-moving module. (Right): The roll motion caused by applying the torque along the $x$ axis of the free-moving module.

The fall off test results unveil better protection from the robot's resilience than the impact test. As the robot is not parallel with the ground during fall off, there are two major contact points of which the first is the robot head, module 00 , and the second is the robot tail, module 11. In Figure 5b, the box chart presents the acceleration of each module between the two major collisions. The rigid robot receives very similar maximum acceleration for all modules of approximately $5400 \mathrm{~m} / \mathrm{s}^{2}$. Module 00 of the tensegrity robot suffers a similar influence as the rigid one from the collision. However, for the rest of the modules, the effect is greatly mitigated at a scale of approximately $80 \%$. As the pretension ratio increases, the maximum acceleration of module 00 slightly decreases due to smaller tension force held inside the structure. The result shows the improved survivability of the robot during a fall off situation to sustain functionality from such accidents.
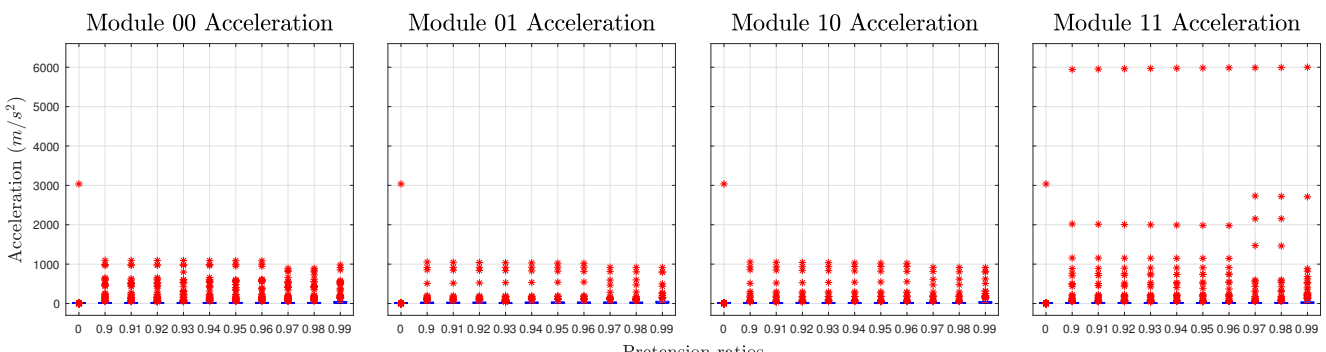

(a)
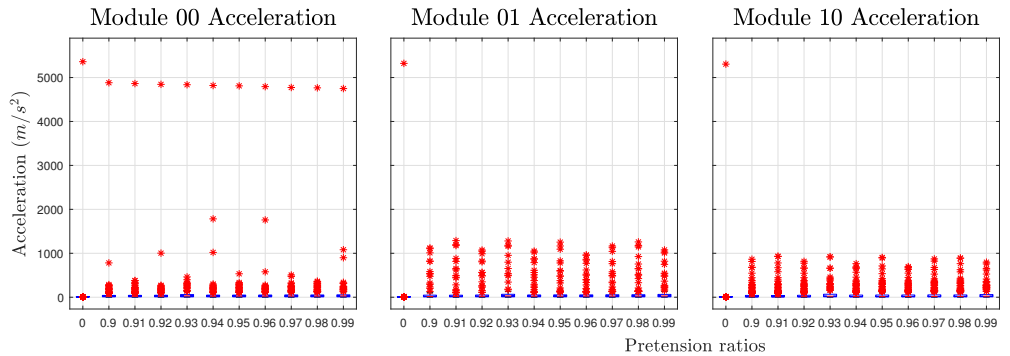

Module 11 Acceleration

(b)

Figure 5. Acceleration box chart of modules after collision. (a) The acceleration of impact test with the ball hitting module 11. (b) The acceleration of fall off test with module 00 contacting the ground first.

\subsection{Locomotion Adaptation}

In Figure 6, the histogram labelled 0 pretension ratio is the contact force of the rigid articulated robot. The active cables of the tensegrity robot are configured with 1.1 times the 
neutral length. Compared with the tensegrity robot, the rigid robot receives much longer zero force bars, especially for wheels 00 and 11, indicating longer improper contact with the ground. It is clear that the rigid robot's wheels bear larger average forces when in contact throughout the process, while for the tensegrity robot the distribution is more flat and lies within a lower range. This reflects a better fit to the terrain of the passively operated tensegrity robot.
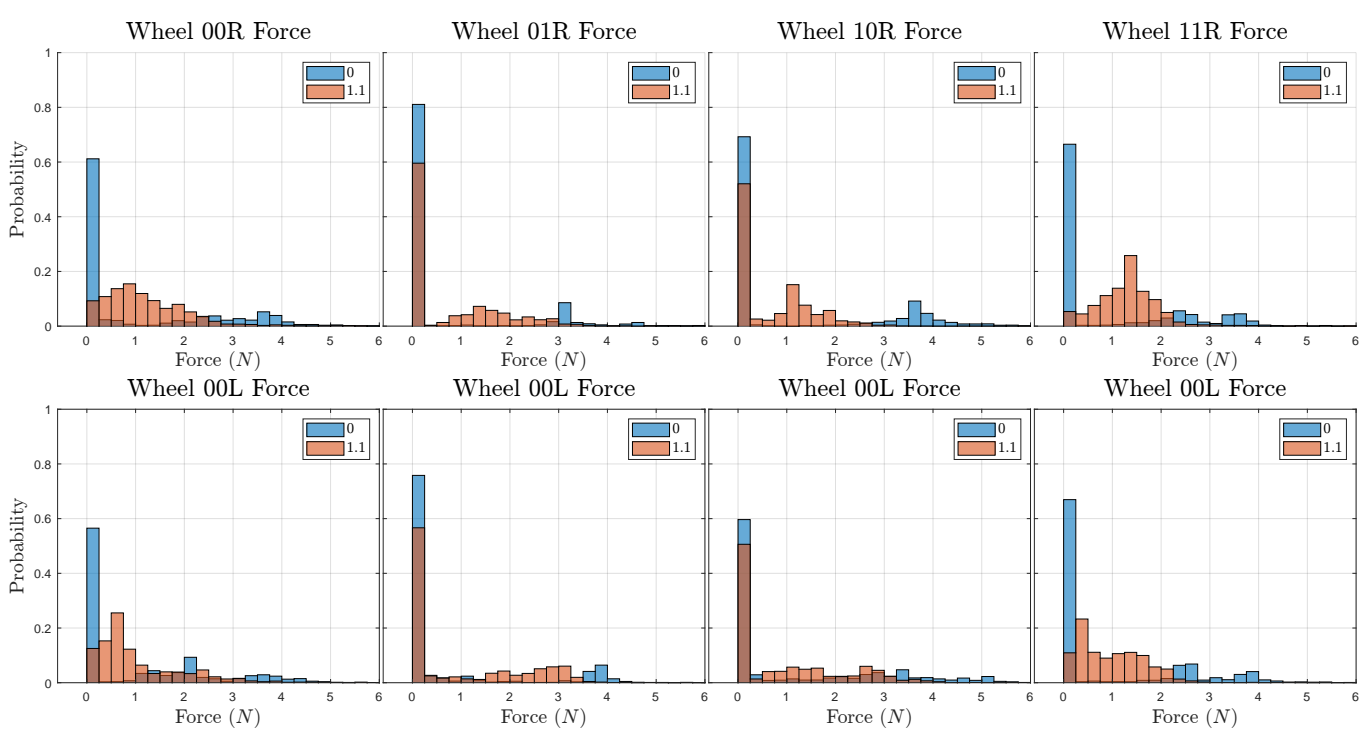

Figure 6. Contact force on the wheels when traversing the same uneven terrain for the tensegrity and rigid robots. 0 represents the rigid robot. 1.1 represents the tensegrity robot with loose active cables of 1.1 times the neutral length.

For active surmounting, testing gives a best result height of $93 \mathrm{~mm}$. Without such active motion, the robot can only cross a step of up to $29 \mathrm{~mm}$ in height. For the robot, each robot module body takes a convex space of a $100 \mathrm{~mm}$ width cube with a wheel diameter of $90 \mathrm{~mm}$. The wheels are attached $40 \mathrm{~mm}$ vertically lower than the centre of gravity of the modules, resulting in the bottom face of the module being $35 \mathrm{~mm}$ above a flat ground. It turns out that the robot is able to surmount an obstacle of $68.9 \%$ of its total height, $103.3 \%$ of its wheel diameter, $265.7 \%$ of its ground clearance, or $320.7 \%$ of the non-actuated passability.

The generated signal of the CPG is plotted in Figure 7a. For each motion's signal, it takes about $1 \mathrm{~s}$ to smoothly start the oscillation. At $9 \mathrm{~s}$, the front assembly's yaw motion is purposely stalled for $1 \mathrm{~s}$. As a result, the other three control signals' frequency is inhibited. After the motion constraint is removed, the oscillators gradually and smoothly restore synchronisation. A reference sinusoidal signal is also plotted for comparison. It is clear that when the synchronisation is established, the CPG output overlaps with the reference.

Self-righting for the two-assembly robot is more difficult than in configurations such as a quadruped as it does not have legs to push off with. The "head" and "tail" are the only contact points available and therefore imitating the behaviour of tortoises to flip over is an efficient solution. To flip by $90^{\circ}$, the manoeuvre is completed by sweeping the head and tail by a quarter of a circle. From the perspective of pitch and yaw motion, it will be a full transit from maximum pitch to maximum yaw or vice versa.

The posture of the robot relative to the world is represented by the front module status with solid lines in Figure $7 \mathrm{~b}$. At the beginning, the roll motion is $90^{\circ}$, indicating a rollover state. Following a step by step sequence of the tensegrity mechanism, which is neutral, full pitch, full pitch and yaw, full yaw and neutral, plotted with dashed lines, the roll posture gradually decreases back to zero, representing a successful self-righting. 


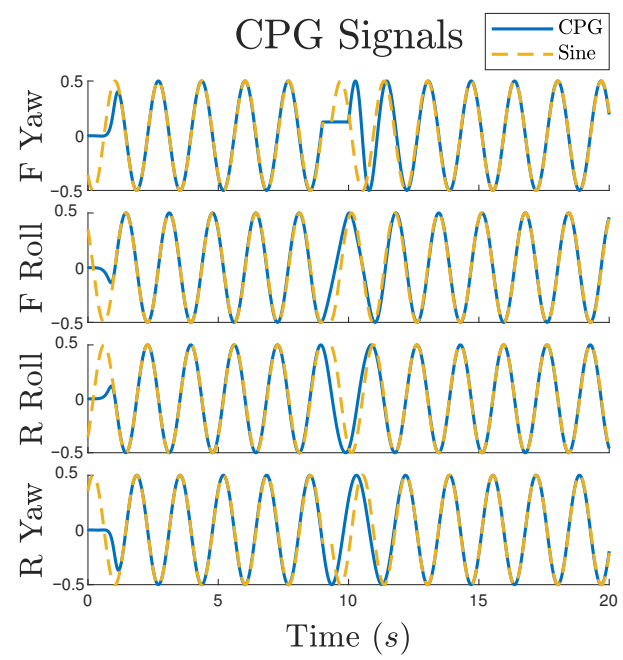

(a)

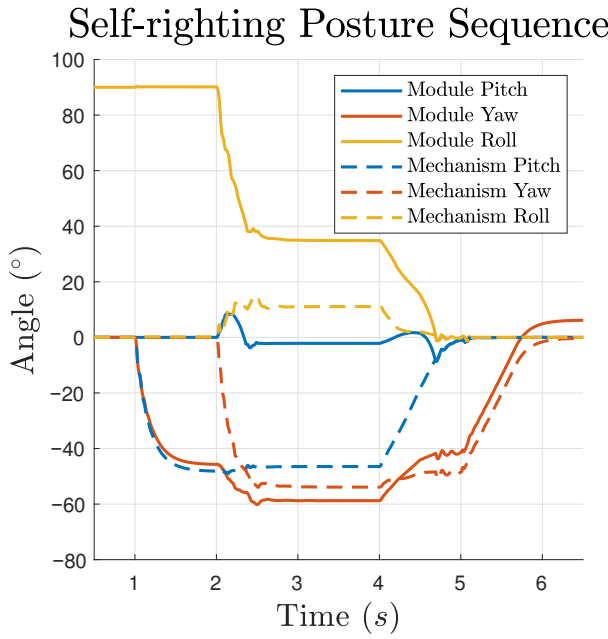

(b)

Figure 7. Waveform of the CPG signal and self-righting posture. (a) The output of each node pair of the CPG network. Two sine wave fragments are plotted with dashed yellow lines for reference. (b) Posture information of one robot assembly. The solid lines denote the orientation of the end module (one not coupled with another assembly). The dashed lines denote the motion of the mechanism.

\section{Discussion}

Based on the results obtained from the various tests with respect to the structure and locomotion, it is clear that introducing a tensegrity structure with sufficient degrees of freedom endows improved resilience to the wheeled modular robot. Compared with soft robots, the proposed tensegrity robot does not make trade-offs on the important criterion of load capacity by taking the form of conventional cuboid modules. Although it cannot be as flexible as a soft robot design, the results indicate obvious resistance capability in terms of collision and unplanned deformation. Additionally, the ability to adopt both wheeled and legged locomotion modes make it advantageous for soft robots. With regard to rigid robots, it is not difficult to realise equivalent degrees of freedom as the proposed tensegrity robot. However, realising a similar rotational flexibility requires compliant-capable rotational actuators to continuously operate in active mode which is power-hungry. On the other hand, as there is no translational compliance in such systems, and the rotational actuators will be at risk of failure with large impacts.

Tensegrity structures need to retain a pretensioned state to preserve their stability and functionality. Although for some tests the robot gives similar performance for different pretension ratios, the static load results indicate good improvement in weight bearing of about $60 \%$ to $80 \%$ with pretension changes. This is beneficial for actuation tasks such as the movement of robots' bodies, legs, and manipulators when overloaded. Compared with existing tensegrity robots that feature a monolithic design, the locomotion of the proposed robot is not limited by the irregular morphology of tensegrity structures. Especially after adopting conventional wheeled locomotion, the efficiency is greatly increased and the robot is no longer affected negatively by the introduction of tensegrity structures. As the robot's assemblies are independently functional and the detachment/attachment of modules is based on the coupling system, the irregularity of tensegrity will not cause side effects in the reconfiguration of the robot as opposed to monolithic morphologies.

It is worth noting that, from observing the results in Figure 4, for each motion, there is an almost linear region extending from the start of applying force. This is counter-intuitive as the displacement is not along the cable's axial direction but rotating around a pivot point with a varying moment arm. To further investigate this, the theoretical force-rotation relationships are calculated, ignoring the small inward displacement of pretension, shown in Figure 8. It can be seen that there are also linear regions in theoretical plots. This is caused by the cancellation of non-linear force curves among the antagonistic cable pairs. 
When all the cables are in a tensioned state, the cables that become longer and the cables that become shorter exhibit a similar trend and result in a linear-like force output. Once the cables that become shorter enter a loose status, the generated force will be dominated by the cables becoming longer and therefore the results enter the non-linear region.
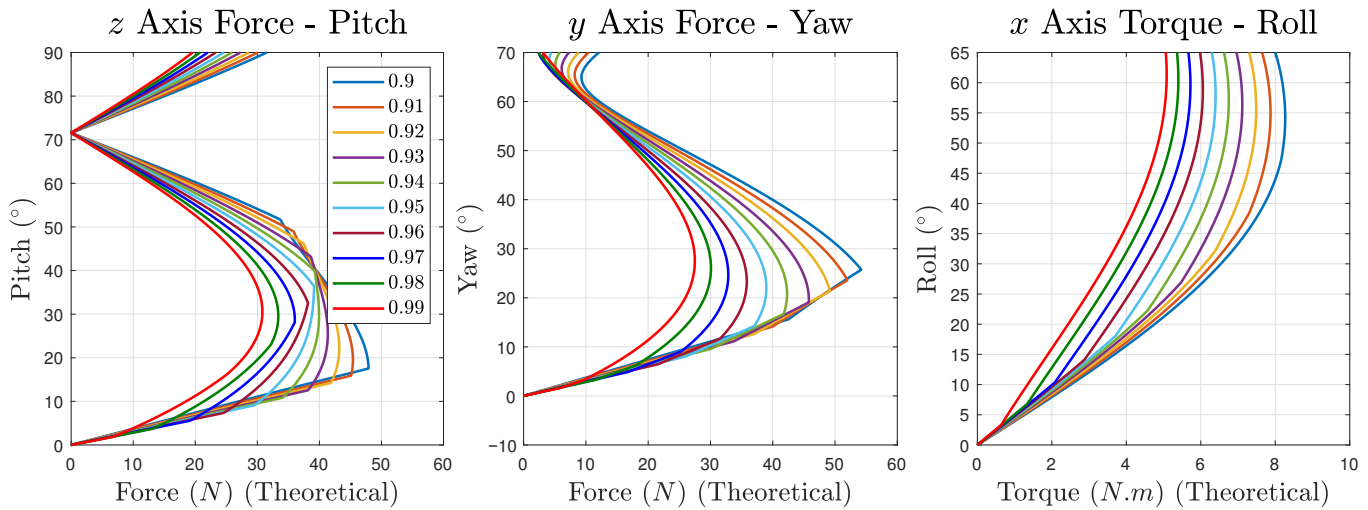

Figure 8. Theoretical force/torque to rotation relationships with the rotation as andependent variable. The horizontal and vertical axis data are swapped to visually match the placement in Figure 4.

It can be observed from Figure $7 \mathrm{~b}$ that the mechanism's pitch and yaw motion curves do not overlap while they actually receive the same target angle. As a sensorless uniform pretension ratio for all actuated cables is used in this paper, the deviation is unavoidable due to flexibility. Fine tuning this is one of the advantages of tensegrity, but the robot requires knowledge of the state. Therefore, one future work direction is to improve the control accuracy of the tensegrity robot by means of either algorithms, feedback, or both.

Although in this paper most of the tests are based on the minimum two-assembly configuration of the robot, its potential should not be limited to this. The three degrees of rotational freedom available actually make each assembly an ideal unit for general purpose actuation tasks inside a comprehensive system. It can be, but is not limited to being, a spine, arm, leg, tail, and manipulator, resulting in morphologies of multiped, snake, or humanoid. These advanced configurations further the capability of the tensegrity robot in harsh environments and form another direction of future work.

\section{Conclusions}

Resilience is a valuable characteristic of robots designed for tough tasks in challenging environments. It is highly linked to the robots' overall survivability and life span. In this paper, a tensegrity robot is presented that is resilient in multiple aspects. In the case of external loads and collisions, the robot demonstrates clear compliance and resistance that can protect it from structural failures. With respect to locomotion, different locomotion modes enabled by the agility of the tensegrity mechanism give the robot flexibility on demand to adapt to different terrains. This allows the robot to avoid or recover from malfunctions in harsh environments. The test results reveal that the characteristics of tensegrity structures are well preserved in such designs while additional agility, mobility, and self-reconfigurability are endowed as well.

The conceptual process of how tensegrity structures can be integrated into a robot instead of transforming a whole robot inspires a new approach for designing tensegrity robots. The proposed wheeled tensegrity modular robot offers a good example of fusing comprehensive resilience into previously rigid robots following such a concept.

In future work, the control accuracy of the tensegrity articulation is a key aspect to be considered. This is expected to be addressed by adding force sensors and using force closure algorithms to calculate the theoretical rest lengths at run-time. Orientation information can also be involved to take posture feedback into account and further improve 
the accuracy. Another direction is to explore the capabilities of different configurations such as quadruped and caterpillar by means of self-reconfiguration.

Author Contributions: Research work, design, testing, results, and writing, T.W.; tensegrity system concept and robotics applications, M.A.P.; supervision and review and editing, M.A.P. and A.M.T. All authors have read and agreed to the published version of the manuscript.

Funding: This research received no external funding.

Institutional Review Board Statement: Not applicable.

Data Availability Statement: The data presented in this study are openly available in Bitbucket at https://bitbucket.org/boring-name/resilience-results.git (accessed on 23 January 2022).

Conflicts of Interest: The authors declare no conflict of interest.

\section{Abbreviations}

The following abbreviations are used in this manuscript:
ABS Acrylonitrile butadiene styrene
CPG Central pattern generator
DoF Degree of freedom
FEA Finite element analysis

\section{References}

1. Tadokoro, S. Rescue robotics challenge. In Proceedings of the 2010 IEEE Workshop on Advanced Robotics and Its Social Impacts, Seoul, Korea, 26-28 October 2010; pp. 92-98.

2. Ellery, A. Environment-robot interaction-The basis for mobility in planetary micro-rovers. Rob. Auton. Syst. 2005, 51, $29-39$. [CrossRef]

3. Miura, H.; Watanabe, A.; Okugawa, M.; Miura, T.; Koganeya, T. Plant inspection by using a ground vehicle and an aerial robot: lessons learned from plant disaster prevention challenge in world robot summit 2018. Adv. Robot. 2020, 34, 104-118. [CrossRef]

4. Post, M.A.; Yan, X.T.; Letier, P. Modularity for the future in space robotics: A review. Acta Astronaut. 2021, 189, 530-547. [CrossRef]

5. Lee, C.; Kim, M.; Kim, Y.J.; Hong, N.; Ryu, S.; Kim, H.J.; Kim, S. Soft robot review. Int. J. Control Autom. Syst. 2017, 15, 3-15. [CrossRef]

6. Marchese, A.D.; Onal, C.D.; Rus, D. Autonomous Soft Robotic Fish Capable of Escape Maneuvers Using Fluidic Elastomer Actuators. Soft Robot. 2014, 1, 75-87. [CrossRef] [PubMed]

7. McMahan, W.; Chitrakaran, V.; Csencsits, M.; Dawson, D.; Walker, I.D.; Jones, B.A.; Pritts, M.; Dienno, D.; Grissom, M.; Rahn, C.D Field trials and testing of the OctArm continuum manipulator. In Proceedings of the 2006 IEEE International Conference on Robotics and Automation, Orlando, FL, USA, 15-19 May 2006; pp. 2336-2341.

8. Deimel, R.; Brock, O. A novel type of compliant and underactuated robotic hand for dexterous grasping. Int. J. Rob. Res. 2016, 35, 161-185. [CrossRef]

9. Polygerinos, P.; Wang, Z.; Galloway, K.C.; Wood, R.J.; Walsh, C.J. Soft robotic glove for combined assistance and at-home rehabilitation. Rob. Auton. Syst. 2015, 73, 135-143. [CrossRef]

10. Zhou, X.; Majidi, C.; O'Reilly, O.M. Soft hands: An analysis of some gripping mechanisms in soft robot design. Int. J. Solids Struct. 2015, 64-65, 155-165. [CrossRef]

11. Choi, C.; Schwarting, W.; DelPreto, J.; Rus, D. Learning Object Grasping for Soft Robot Hands. IEEE Robot. Autom. Lett. 2018, 3, 2370-2377. [CrossRef]

12. Shah, D.S.; Booth, J.W.; Baines, R.L.; Wang, K.; Vespignani, M.; Bekris, K.; Kramer-Bottiglio, R. Tensegrity Robotics. Soft Robot. 2021, ahead of print. [CrossRef]

13. Buckminster Fuller, R.; Marks, R. The Dymaxion World of Buckminster Fuller; Anchor Books: Garden City, NY, USA, 1973.

14. Skelton, R.E.; William Helton, J.; Adhikari, R.; Pinaud, J.P.; Chan, W. An Introduction to the Mechanics of Tensegrity Structures. In The Mechanical Systems Design Handbook: Modeling, Measurement, and Control; Hurmuzlu, Y., Nwokah, O.D.I., Eds.; CRC Press: Boca Raton, FL, USA, 2002.

15. Komendera, E. A Survey of the Computational Modeling and Control of Tensegrity Robots. Ph.D. Thesis, University of Colorado, Boulder, CO, USA, 2012.

16. Tibert, A.G.; Pellegrino, S. Review of Form-Finding Methods for Tensegrity Structures. Int. J. Space Struct. 2003, 18, $209-223$. [CrossRef]

17. Bruce, J.; Sabelhaus, A.P.; Chen, Y.; Lu, D.; Morse, K.; Milam, S.; Caluwaerts, K.; Agogino, A.M.; Sunspiral, V. SUPERball: Exploring Tensegrities for Planetary Probes. In Proceedings of the International Symposium on Artificial Intelligence, Robotics and Automation in Space (i-SAIRAS), Montreal, QC, Canada, 17-19 June 2014. 
18. Sabelhaus, A.P.; Bruce, J.; Caluwaerts, K.; Chen, Y.; Lu, D.; Liu, Y.; Agogino, A.K.; SunSpiral, V.; Agogino, A.M. Hardware Design and Testing of SUPERball, A Modular Tensegrity Robot. In Proceedings of the 2014 Edition of the World Conference on Structural Control and Monitoring (WCSCM), Barcelona, Spain, 15-17 July 2014.

19. Bruce, J.; Caluwaerts, K.; Iscen, A.; Sabelhaus, A.P.; SunSpiral, V. Design and evolution of a modular tensegrity robot platform. In Proceedings of the 2014 IEEE International Conference on Robotics and Automation (ICRA), Hong Kong, China, 31 May-7 June 2014; pp. 3483-3489.

20. Sabelhaus, A.P.; Bruce, J.; Caluwaerts, K.; Manovi, P.; Firoozi, R.F.; Dobi, S.; Agogino, A.M.; SunSpiral, V. System design and locomotion of SUPERball, an untethered tensegrity robot. In Proceedings of the 2015 IEEE International Conference on Robotics and Automation (ICRA), Seattle, WA, USA, 26-30 May 2015; pp. 2867-2873.

21. Bruce, J. Design, Building, Testing, and Control of SUPERball: A Tensegrity Robot to Enable New Forms of Planetary Exploration. Ph.D. Thesis, University of California, Santa Cruz, CA, USA, 2016.

22. Vespignani, M.; Friesen, J.M.; SunSpiral, V.; Bruce, J. Design of SUPERball v2, a Compliant Tensegrity Robot for Absorbing Large Impacts. In Proceedings of the 2018 IEEE/RSJ International Conference on Intelligent Robots and Systems (IROS), Madrid, Spain, 1-5 October 2018; pp. 2865-2871.

23. Kim, K.; Chen, L.; Cera, B.; Daly, M.; Zhu, E.; Despois, J.; Agogino, A.K.; SunSpiral, V.; Agogino, A.M. Hopping and rolling locomotion with spherical tensegrity robots. In Proceedings of the 2016 IEEE/RSJ International Conference on Intelligent Robots and Systems (IROS), Daejeon, Korea, 9-14 October 2016; pp. 4369-4376.

24. Zha, J.; Wu, X.; Kroeger, J.; Perez, N.; Mueller, M.W. A collision-resilient aerial vehicle with icosahedron tensegrity structure. In Proceedings of the 2020 IEEE/RSJ International Conference on Intelligent Robots and Systems (IROS), Las Vegas, NV, USA, 24 October-24 January 2020; pp. 1407-1412.

25. Zhou, D.; Liu, Y.; Tang, X.; Sun, J.; Deng, J. A Lightweight and Multi-Motion Crawling Tensegrity Robot Driven by Twisted Artificial Muscles. IEEE Trans. Ind. Electron. 2021, early access. [CrossRef]

26. Tietz, B.R.; Carnahan, R.W.; Bachmann, R.J.; Quinn, R.D.; SunSpiral, V. Tetraspine: Robust terrain handling on a tensegrity robot using central pattern generators. In Proceedings of the 2013 IEEE/ASME International Conference on Advanced Intelligent Mechatronics, Wollongong, Australia, 9-12 July 2013; pp. 261-267.

27. Friesen, J.M.; Glick, P.; Fanton, M.; Manovi, P.; Xydes, A.; Bewley, T.; Sunspiral, V. The second generation prototype of a Duct Climbing Tensegrity robot, DuCTTv2. In Proceedings of the IEEE International Conference on Robotics and Automation, Stockholm, Sweden, 16-21 May 2016; pp. 2123-2128.

28. Rieffel, J.; Mouret, J.B. Adaptive and Resilient Soft Tensegrity Robots. Soft Robot. 2018, 5, 318-329. [CrossRef]

29. Liu, K.; Paulino, G.H. Tensegrity topology optimization by force maximization on arbitrary ground structures. Struct. Multidiscip. Optim. 2019, 59, 2041-2062. [CrossRef]

30. Chen, L.; Cera, B.; Zhu, E.L.; Edmunds, R.; Rice, F.; Bronars, A.; Tang, E.; Malekshahi, S.R.; Romero, O.; Agogino, A.K.; et al Inclined surface locomotion strategies for spherical tensegrity robots. In Proceedings of the 2017 IEEE/RSJ International Conference on Intelligent Robots and Systems (IROS), Vancouver, BC, Canada, 24-28 September 2017; pp. 4976-4981.

31. Mirletz, B.T.; Park, I.W.; Flemons, T.E.; Sunspiral, V. Design and Control of Modular Spine-Like Tensegrity Structures In Proceedings of the 6th World Conference on Structural Control and Monitoring, Barcelona, Spain, 15-17 July 2014.

32. Hustig-Schultz, D.; SunSpiral, V.; Teodorescu, M. Morphological design for controlled tensegrity quadruped locomotion. In Proceedings of the 2016 IEEE/RSJ International Conference on Intelligent Robots and Systems (IROS), Daejeon, Korea, 9-14 October 2016; pp. 4714-4719.

33. Böhm, V.; Zimmermann, K. Vibration-driven mobile robots based on single actuated tensegrity structures. In Proceedings of the 2013 IEEE International Conference on Robotics and Automation, Karlsruhe, Germany, 6-10 May 2013; pp. 5475-5480.

34. Caluwaerts, K.; Despraz, J.; Işçen, A.; Sabelhaus, A.P.; Bruce, J.; Schrauwen, B.; SunSpiral, V. Design and control of compliant tensegrity robots through simulation and hardware validation. J. R. Soc. Interface 2014, 11, 20140520. [CrossRef]

35. Carreño, F.; Post, M.A. Design of a novel wheeled tensegrity robot: A comparison of tensegrity concepts and a prototype for travelling air ducts. Robot. Biomim. 2018, 5, 1-24. [CrossRef]

36. Biswal, P.; Mohanty, P.K. Development of quadruped walking robots: A review. Ain Shams Eng. J. 2021, 12, 2017-2031. [CrossRef]

37. Wang, T.; Post, M.A. A Symmetric Three Degree of Freedom Tensegrity Mechanism with Dual Operation Modes for Robot Actuation. Biomimetics 2021, 6, 30. [CrossRef] [PubMed]

38. Wang, F.; Qian, Z.; Yan, Z.; Yuan, C.; Zhang, W. A Novel Resilient Robot: Kinematic Analysis and Experimentation. IEEE Access 2020, 8, 2885-2892. [CrossRef]

39. Ramachandran, R.K.; Preiss, J.A.; Sukhatme, G.S. Resilience by Reconfiguration: Exploiting Heterogeneity in Robot Teams In Proceedings of the 2019 IEEE/RSJ International Conference on Intelligent Robots and Systems (IROS), Macau, China, 3-8 November 2019; pp. 6518-6525.

40. Zhang, W.J.; Lin, Y. On the principle of design of resilient systems-Application to enterprise information systems. Enterp. Inf. Syst. 2010, 4, 99-110. [CrossRef]

41. Post, M.A.; Austin, J. Knowledge-Based Self-Reconfiguration And Self-Aware Demonstration For Modular Satellite Assembly. In Proceedings of the 10th International Workshop on Satellite Constellations \& Formation Flying, Glasgow, UK, 16-19 July 2019.

42. Ijspeert, A.J.; Crespi, A.; Ryczko, D.; Cabelguen, J.M. From swimming to walking with a salamander robot driven by a spinal cord model. Science 2007, 315, 1416-1420. [CrossRef] [PubMed] 